\title{
Management of the inter-segmental plane using the "Combined Dimensional Reduction Method" is safe and viable in uniport video-assisted thoracoscopic pulmonary segmentectomy
}

\author{
Bin Zheng ${ }^{1}$, Guobing Xu${ }^{1}$, Xiayu Fu ${ }^{1}$, Weidong $W^{1}{ }^{1}$, Mingqiang Liang ${ }^{1}$, Taidui Zeng ${ }^{1}$, Shuliang Zhang ${ }^{1}$, \\ Yong Zhu ${ }^{1}$, Wei Zheng ${ }^{1}$, Chun Chen ${ }^{1}$, Benoît Bédat ${ }^{2}$, Scott J. Swanson ${ }^{3}$, Terumoto Koike ${ }^{4}$, \\ Hisashi Iwata $^{5}$, Benedetta Bedetti ${ }^{6}$, Masaaki Sato ${ }^{7}$; written on behalf of the AME Thoracic Surgery \\ Collaborative Group
}

${ }^{1}$ Thoracic Department, Fujian Medical University Union Hospital, Fuzhou 350001, China; ${ }^{2}$ Thoracic and Endocrine Unit, University Hospitals of Geneva, Geneva, Switzerland; ${ }^{3}$ Division of Thoracic Surgery, Brigham and Women's Hospital, Harvard Medical School, Boston, USA; ${ }^{4}$ Division of Thoracic and Cardiovascular Surgery, Niigata University Graduate School of Medical and Dental Sciences, Niigata, Japan; ${ }^{5}$ Department of General Thoracic Surgery, Center of Respiratory Disease, Gifu University Hospital, Gifu, Japan; ${ }^{6}$ Department of Thoracic Surgery, Malteser Hospital, Bonn, Germany; ${ }^{7}$ Department of Thoracic Surgery, The University of Tokyo Hospital, Bunkyo, Tokyo, Japan

Contributions: (I) Conception and design: B Zheng, G Xu, Y Zhu, W Zheng, C Chen; (II) Administrative support: B Zheng, Y Zhu, W Zheng, C Chen; (III) Provision of study materials or patients: B Zheng, Y Zhu, W Zheng, C Chen; (IV) Collection and assembly of data: B Zheng, G Xu, X Fu, W Wu, M Liang, T Zeng, S Zhang; (V) Data analysis and interpretation: B Zheng, G Xu, X Fu, W Wu, M Liang, T Zeng, S Zhang; (VI) Manuscript writing: All authors; (VII) Final approval of manuscript: All authors.

Correspondence to: Chun Chen, MD. Thoracic Department, Fujian Medical University Union Hospital, No. 29, Xinquan Road, Fuzhou 350001, China. Email: lacustrian@163.com.

Background: The management of the intersegmental plane (ISP) is challenging during uniport videoassisted thoracoscopic (VATS) pulmonary segmentectomy. Staplers and electrocautery have been used extensively in ISP management. However, both of them have their respective drawbacks. Currently, we have provided a revised technique termed as "Combined Dimensional Reduction Method" (CDR method), for managing the ISP with combined application of ultrasonic scalpel and staplers. The study aimed to review the outcomes of patients who underwent uniport VATS segmentectomy with or without the CDR method in our institute and assess the feasibility and safety of the CDR method.

Methods: From March 2017 to February 2018, 220 patients who underwent uniport VATS segmentectomy were retrospectively reviewed. By using IQQA software, pulmonary structures were reconstructed as three-dimensional (3D) images, making the targeted structures could be identified preoperatively. For the management of the ISP, in the CDR group, we firstly used the ultrasonic scalpel to trim the 3D pulmonary structure along the intersegmental demarcation, making the remaining targeted parenchyma both sufficiently thin enough and located on a 2D plane; thus, enabling easy use of staplers in managing ISP. Whereas, in the non-CDR group, we only use the staplers to manage the ISPs. The clinical characteristics, complications, and postoperative pulmonary functions were compared between the two groups.

Results: Propensity score analysis generated 2 well-matched pairs of 71 patients in CDR and non-CDR groups. There was no 30-day postoperative death or readmission in either group. The CDR group was significantly associated with the shorter operative time $(178.3 \pm 35.8$ vs. $209.2 \pm 28.7 \mathrm{~min})(\mathrm{P}=0.031)$ and postoperative stay $(4.5 \pm 2.3$ vs. $5.7 \pm 4.2$ days) $(\mathrm{P}=0.041)$, compared to the non-CDR group. Moreover, no significant difference was observed in blood loss, a period of chest tube drainage, a period of ultrafine tube drainage, and postoperative pulmonary complications between the two groups. Moreover, the recovery rate of postoperative forced expiratory volume in 1 second (FEV1) or vital capacity (VC) at 1 and 3 months after segmentectomy was comparable between them.

Conclusions: The CDR method could make segmentectomy easier and more accurate, and therefore has the potential to be a viable and effective technique for uniport VATS pulmonary segmentectomy. 
Keywords: Intersegmental plane (ISP); segmentectomy; uniport video-assisted thoracoscopic (uniport VATS); ultrasonic scalpel; staplers

Submitted Mar 03, 2019. Accepted for publication Aug 08, 2019.

doi: $10.21037 /$ tlcr.2019.09.12

View this article at: http://dx.doi.org/10.21037/tlcr.2019.09.12

\section{Introduction}

Computer tomography (CT) screening increases the incidence of finding early-stage lung cancer $(1,2)$. Uniport video-assisted thoracoscopic (VATS) segmentectomy is a parenchyma-sparing technique, considered an effective treatment for early-stage lung cancer, metastases, and benign lesions. However, VATS segmentectomy can be a challenging procedure, as the identification of the segmental structures and the management of intersegmental plane (ISP) is more complicated than in a standard VATS lobectomy. Several methods have been reported for the management of ISP. Some surgeons prefer using staplers, although this practice can shrink the preserved segments and result in insufficient reexpansion. Electrocautery has also been shown to be useful for dissecting the ISP, especially for central lesions $(3,4)$; however, eschars caused by the application of electrocautery make it more difficult to distinguish the ISP, and the use of electrocautery on the lung causes a higher incidence of postoperative air leak $(3,5)$.

Therefore, we have provided a revised technique termed "Combined Dimensional Reduction Method" (CDR method) for uniport VATS segmentectomy, which entails combining the application of ultrasonic scalpel and staplers for the management of ISP. The study aimed to review the results of patients who underwent uniport VATS segmentectomy with or without the CDR method in our institute and to assess the feasibility and safety of the CDR method.

\section{Methods}

\section{Patients}

From March 2017 to February 2018, 220 patients who underwent uniport VATS segmentectomy were retrospectively reviewed. The inclusion criteria of patient cases were as follows: (I) indication for surgery was discussed in a multidisciplinary team; (II) informed consent obtained from the patients after discussion of the general risks and benefits of pulmonary segmentectomy. The exclusion criteria of patient cases were the following: (I) age over 75 years old; (II) history of pulmonary tuberculosis or other inflammatory thoracic diseases which could cause severe adhesions; (III) underwent preoperative chemotherapy or radiotherapy; (IV) patients lost to follow-up. Eventually, 189 patients met the selection criteria and were enrolled in our study.

A propensity score matching analysis was conducted to reduce any possible bias regarding preoperative characteristics. The propensity scores were generated using a logistic regression method based on clinically relevant variables such as age, gender, smoking pack years and predicted forced expiratory volume in 1 second (FEV1), which were considered as potential associations with the outcome of interest based on clinical knowledge. After matching, there were 71 patients in the CDR group (segmentectomy with CDR method) and 71 in the nonCDR group (segmentectomy without CDR method). The ethics committee approved this study of Fujian Medical University Union Hospital.

\section{Artificial intelligence-assisted mapping planning}

CT images were obtained using 64-slice spiral CT scanner GE Light Speed VCT (GE Healthcare, CT, USA) with a slice thickness of $1.25 \mathrm{~mm}$. Both non-enhanced and contrast-enhanced scans were performed. CT data of all patients were stored in the DICOM format. In both groups, the data for all patients were imported in the IQQAGuide (EDDA Technology, Princeton, NJ, USA). The software automatically recognizes the pulmonary structures and reconstructs the three-dimensional (3D) pulmonary structures of the bronchial tree, arteries, and veins. The small number of differences between the reconstructed structures and two-dimensional (2D) CT images were manually reconciled. After reconstruction, the data were saved as still images and dynamic videos. Measured 2D data, including diameters of lesions and structures, along with lengths and the angles of branches of vessels and bronchi, were determined by measurement tools in IQQA. The 


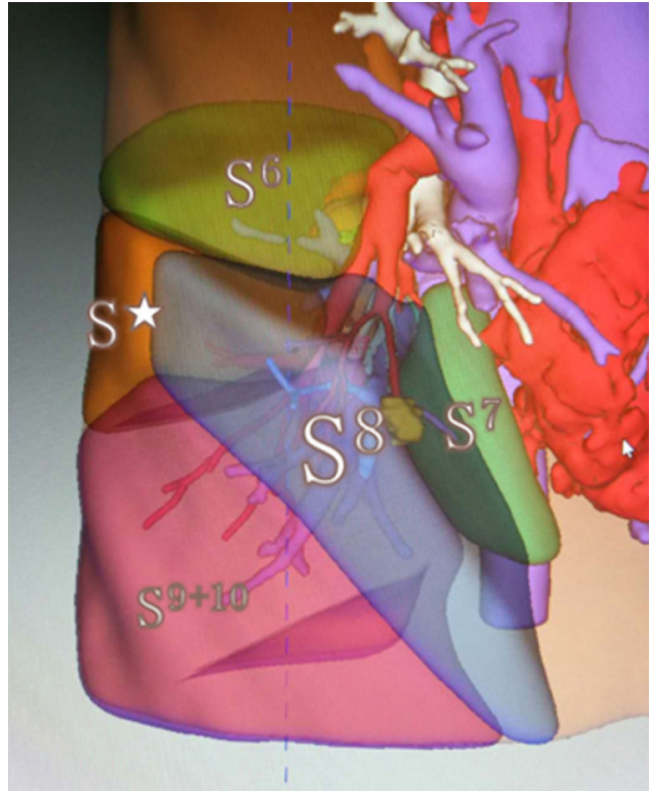

Figure 1 Virtual surgery was performed on the reconstructed dynamic 3D model (take RS8 as an example). All 2D data of segmental structures were analyzed, including the location, length, number of branches, diameter of branches, and angle of branches. $\mathrm{S}^{*}$ means subsuperior segment.

positional relationship between lesions and the segmental structures were defined, and the possible variant segmental structures were analyzed. Virtual surgery was performed on the reconstructed dynamic 3D model (Figure 1), and the remaining pulmonary volume was calculated to evaluate the feasibility of the proposed operation strategies. The treatment plan was finally defined after optimization of the individualized virtual surgery.

\section{Surgical procedures}

In this study, all of the surgeries were performed by the same group of surgeons. After endotracheal intubation with either a left- or right-sided double-lumen endotracheal tube, the affected lung was deflated as soon as the thoracic cavity was opened. Pulmonary segmentectomy was performed via uniportal thoracoscopy incision $(3.5$ to $4 \mathrm{~cm}$ long). During segmentectomy, we did not routinely palpate the lung to find the nodule. Instead, the targeted segment bronchi, arteries, and veins were directly identified according to the artificial intelligence-assisted mapping planning. Targeted segmental structures were dissected by ligation or stapler cutting. Finally, the deflated lung was inflated completely with controlled airway pressure with pure oxygen under the pressure of $20 \mathrm{cmH} 2 \mathrm{O}$, with the bronchus of the operation side open to the atmosphere while continuing the ventilation of the contralateral lung $(6,7)$. Ten to fifteen minutes later, a demarcation line developed between the inflated targeted segment and the deflated adjacent segments, with the inflated-deflated line representing the intersegmental border.

Regardless of segmentectomy type, the ISPs were all presented as $3 \mathrm{D}$ structures. In the CDR group, according to the guidance of intersegmental deflation-inflation demarcation, we firstly used an ultrasonic scalpel to cut the intersegmental parenchyma from the hilum to the distal part, lifting the bronchial stump away from the hilum, finishing nearly three-fourths of the proximal parenchyma division, and making the remaining peripheral parenchyma of the targeted segment thin enough (Figure 2A). In this way, the staplers could thus be quickly orientated in the resection plane to cut the remaining parenchyma (Figure $2 B$ ), whereas, in the non-CDR group, we use the staplers only to manage the ISPs.

An adequate surgical margin was controlled during segmentectomy. We defined the adequate margin as the resection margin $\geq 2 \mathrm{~cm}$ or the ratio of (resection margin/ tumor diameter) $\geq 1$. If the margin was not enough, additional resection of the adjacent segment or subsegment was performed.

\section{CDR method protocols}

In the CDR method, the "combine" term describes two components: (I) the preoperative IQQA reconstruction together with intraoperative guidance and confirmation for precise segmentectomy; and (II) the combined application of ultrasonic scalpel and staplers to the management of ISP during surgery. Similarly, "dimensional reduction" also has two components. (I) 2D measured data from CT scan, including nodule locations, number of branches, angle of branches, and lengths of branches, as determined by measurement tools of $3 \mathrm{D}$ software. (II) The use of an ultrasonic scalpel to perform the plasticity, making the remaining targeted parenchyma both sufficiently thin enough and located on a 2D plane, thus enabling easy use of staplers in managing ISP, especially in uniportal operations.

\section{Postoperative management protocols}

All patients were extubated in theater and transferred 

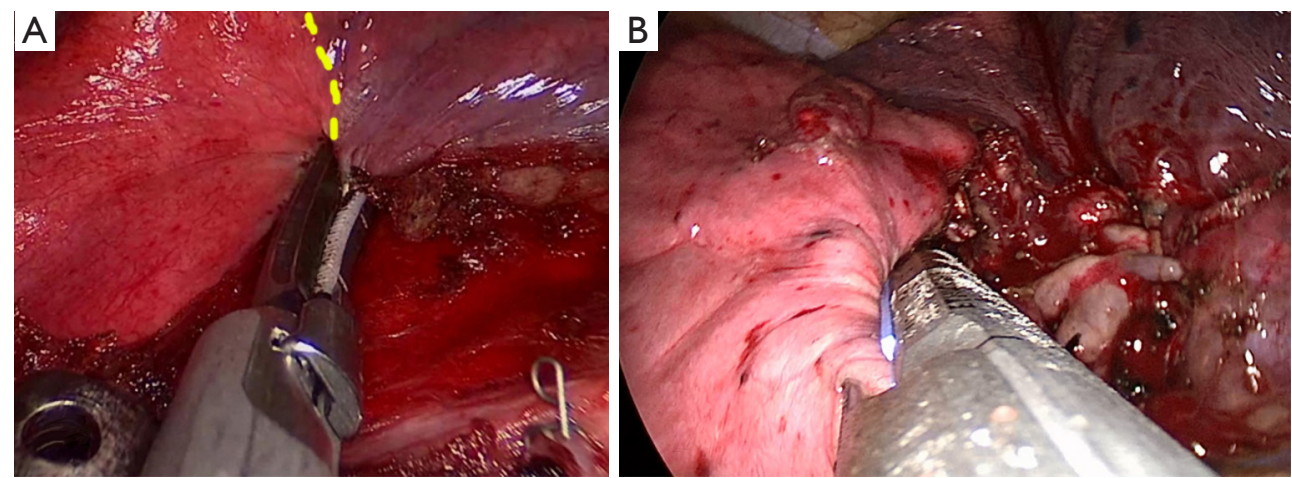

Figure 2 Intersegmental plane management procedures of Combined Dimensional Reduction Method. (A) Ultrasonic scalpel was used to perform the plasticity of pulmonary parenchyma along the intersegmental plane, making remaining targeted parenchyma sufficiently thin and located on a 2D plane. (B) Staplers were used to do the cutting of the remaining parenchyma according to the intersegmental plane.

to the general ward. A chest tube (28 Fr) was used as an upper drainage tube, and an ultrafine chest drainage tube (ABLE, 8 Fr, Guangdong Baihe Medical Technology Co., Guangdong, China) was set as the lower tube. Generally, withdrawal criterion of the upper chest tube was considered to be the absence of air leak through the tube, and after removing the upper tube, we commonly left the ultrafine tube in place for drainage of pleural effusion. The day after chest tube withdrawal, a chest $\mathrm{x}$-ray was performed to detect for a pneumothorax. The ultrafine chest tube was removed when the pleural fluid drainage was under $200 \mathrm{~mL}$ per day.

If all conditions were met, meaning no complications occurred in the postoperative period, a chest X-ray showed no pneumothorax, severe pleural effusion or pulmonary infection, and the patients had good ambulation, then the patients were discharged.

\section{Follow up}

Patients were scheduled for follow-up examinations at our out-patient department in 1 and 3 months after surgery, and then every 3 months for the first 2 years following surgery. During follow-up, patients underwent the following examinations: medical history, physical examination, chest radiography, neck-abdomen CT scans, and abdominal ultrasonography. The pulmonary function test was measured at 1 and 3 months postoperatively. Moreover, the bronchoscope, brain MR, bone scan, or PET-CT was conducted if indicated.

\section{Data analysis and statistics}

Propensity score matching was conducted to reduce the bias of the study and control the potential differences in the preoperative characteristics of patients in both groups. The propensity scores were calculated, using a logistic regression based on clinically relevant preoperative variables, including age, gender, amount of packs smoked per year, and predicted FEV1. Patients were matched 1:1 by the nearest neighbor matching without replacement.

The outcomes of interest in our study were the operation time, blood loss of the operation, period of chest tube drainage, the period of ultrafine tube drainage, postoperative stay, postoperative pulmonary complications, and the recovery rate of postoperative FEV1 and vital capacity (VC). Operation time was defined as the time from skin incision to skin closure. Blood loss was defined as the bleeding volume during surgery. Period of chest tube drainage defined as the date from surgery to remove the chest tube. Period of ultrafine tube drainage defined as the date from surgery to remove the ultrafine tube. Postoperative stay defined as the date from surgery to discharge. Postoperative pulmonary complications mainly consisted of pulmonary infection, delayed pneumothorax, and prolonged air leak. The pulmonary infection required positive sputum cultures or clear clinical and radiographic evidence of consolidation. A prolonged air leak was defined as air leakage lasting 7 days or more. Delayed pneumothorax was defined as pneumothorax inclusive of increasing residual dead space on the surgical side after 
Table 1 Clinical characteristics of patients in the non-CDR and CDR groups

\begin{tabular}{|c|c|c|c|}
\hline Characteristics & Non-CDR group ( $\mathrm{n}=71), \mathrm{n}(\%)$ & CDR group $(n=71), n(\%)$ & $\mathrm{P}$ \\
\hline Sex & & & 0.077 \\
\hline Male & $19(26.8)$ & $29(40.8)$ & \\
\hline Female & $52(73.2)$ & $42(59.2)$ & \\
\hline Types of segmentectomy & & & 0.867 \\
\hline Segmentectomy & $36(50.7)$ & $40(56.3)$ & \\
\hline Multi-segmentectomy & $29(40.8)$ & $19(26.8)$ & \\
\hline Subsegmentectomy & $1(1.4)$ & $6(8.5)$ & \\
\hline Adenocarcinoma & $56(78.9)$ & $64(90.1)$ & \\
\hline Other malignancy & $2(2.8)$ & $1(1.4)$ & \\
\hline Benign nodule & $13(18.3)$ & $6(8.5)$ & \\
\hline
\end{tabular}

CDR, Combined Dimensional Reduction Method; SD, standard deviation.

chest tube removal. A pulmonary function test was carried out with a spirometer (CHESTAC-8800, CHEST M.I., Tokyo, Japan) in our hospital. The FEV1 and VC were measured at 1 and 3 months postoperatively. Predicted postoperative pulmonary function was calculated according to the previously reported formula (8). The actual degree of postoperative functional loss in proportion to the resected lung volume was calculated as the recovery rate, by the following formula: (measured postoperative value/predicted postoperative value) $\times 100 \%$.

JMP 12.2.0 software (SAS Institute, Inc., Cary, NC, USA) was used for statistical evaluations. Data between groups were compared, using the Student $t$-test, Pearson $\chi^{2}$ test, Fisher's exact test or the 2-sample rank-sum test, if appropriate. In all comparisons, 2 -tailed tests were performed. Differences between groups were considered significant when $\mathrm{P}$ was less than 0.05 . The continuous data were expressed as mean \pm standard deviation (SD). Categorical data were expressed as counts and proportions.

\section{Results}

Propensity score analysis generated 2 well-matched pairs of 71 patients in both groups. The clinical characteristics of the study population $(n=142)$ are summarized in Table 1. In the CDR group, there were 42 female and 29 male patients, with a mean age of 52.2 years. There were 40 , 19 , and 6 patients underwent segmentectomy, multisegmentectomy, and subsegmentectomy, respectively, and most of the cases were adenocarcinoma ( $n=64,90.1 \%)$. In regards to the non-CDR group, which included 52 women and 19 men, there were 36, 29, and 1 patient underwent segmentectomy, multi-segmentectomy, and subsegmentectomy, respectively. Similarly, most of the cases were adenocarcinoma $(n=56,78.9 \%)$. There was no significant difference in the observed variables of clinical characteristics, such as age, gender, tumor size, type of segmentectomy, and pathological diagnosis. The locations of the resected pulmonary segments are summarized in Table 2. Resected segment groups included segmentectomy, multi-segmentectomy, subsegmentectomy, along with other kinds of segmentectomy. Right $S^{1}$, right $S^{6}$, upper division, and lingular division were the most commonly resected segments.

The operative characteristics are summarized in Table 3, including the operation time, blood loss of the operation, period of chest tube drainage, a period of ultrafine tube drainage, postoperative stay, and postoperative pulmonary 
Table 2 Location of resected segments

\begin{tabular}{|c|c|c|c|}
\hline Locations & $\begin{array}{l}\text { Non-CDR group } \\
\qquad(\mathrm{n}=71)\end{array}$ & $\begin{array}{l}\text { CDR group } \\
\quad(n=71)\end{array}$ & Total \\
\hline \multicolumn{4}{|l|}{ Right upper } \\
\hline$S^{1}$ & 5 & 11 & 16 \\
\hline$S^{2}$ & 6 & 4 & 10 \\
\hline$S^{1}+S^{2}$ & 7 & 5 & 12 \\
\hline $\mathrm{S}^{3}$ & 4 & 6 & 10 \\
\hline$S^{3 b}$ & 0 & 1 & 1 \\
\hline$S^{1}+S^{3}$ & 1 & 1 & 2 \\
\hline \multicolumn{4}{|l|}{ Right lower } \\
\hline$S^{6}$ & 11 & 9 & 20 \\
\hline$S^{7}$ & 1 & 0 & 1 \\
\hline$S^{8}$ & 3 & 1 & 4 \\
\hline$S^{9}$ & 1 & 0 & 1 \\
\hline \multicolumn{4}{|l|}{ Left upper } \\
\hline$S^{1+2}$ & 4 & 7 & 11 \\
\hline$S^{1+2 a+b}$ & 1 & 4 & 5 \\
\hline$S^{1+2 a+b+S 3}$ & 0 & 1 & 1 \\
\hline $\mathrm{S}^{3}$ & 3 & 2 & 5 \\
\hline Upper division & 10 & 7 & 17 \\
\hline Lingular division & 7 & 6 & 13 \\
\hline \multicolumn{4}{|l|}{ Left lower } \\
\hline$S^{6}$ & 5 & 0 & 5 \\
\hline$S^{8}$ & 2 & 4 & 6 \\
\hline$S^{9}$ & 0 & 2 & 2 \\
\hline
\end{tabular}

CDR, Combined Dimensional Reduction Method.

complications. The operative time in the CDR group was shorter than that in the non-CDR group $(178.3 \pm 35.8$ vs. $209.2 \pm 28.7 \mathrm{~min})(\mathrm{P}=0.031)$. Postoperative stay in the CDR group was shorter than that in the non-CDR group $(4.5 \pm 2.3$ vs. $5.7 \pm 4.2$ days $)(\mathrm{P}=0.041)$. There was no significant difference in blood loss, a period of chest tube drainage, a period of ultrafine tube drainage, and postoperative pulmonary complications. Pulmonary infection was observed in 7 patients (4.9\%), 3 of whom were patients in the non-CDR group, and 4 of whom were patients in the CDR group. Two patients had a delayedonset pneumothorax (1.4\%), with 1 patient in the non-
CDR group, and 1 patient in the CDR group. Two patients needed re-chest-drainage, 1 patient for 2 days, and the other for 5 days. Two patients had prolonged air leaks (1.4\%), with 1 patient in the non-CDR group, and 1 patient in the CDR group. Two patients had chest drainage, 1 patient's lasting for 12 days, and the other's last for 8 days. Other postoperative complications happened in 2 patients, including liver dysfunction in 1 patient in the non-CDR group and severe arrhythmia in 1 patient in the CDR group. There was no 30-day postoperative death or readmission in either group, and the recovery rate of postoperative FEV1 or VC at 1 and 3 months after segmentectomy was comparable between them (Figure 3).

\section{Discussion}

In recent years, more and more evidence has suggested that anatomic pulmonary segmentectomy appears to be a valid alternative to VATS lobectomy in patients with early-stage non-small cell lung cancer (9-14). However, the location of the nodule, precise resection, and management of ISP remain significant challenges for thoracic surgeons.

Several techniques to locate the nodule for a better resection were evaluated. Besides palpation of small nodules during segmentectomy, there are several other invasive marking techniques such as marking using a hook wire, using various dyes or special agents $(15,16)$. These techniques have many limitations, including diffusion of the dyes, complications including pneumothorax, hemorrhage, dislodgement, and even fatal air embolisms $(15,17,18)$. Multiple segments are often involved in the growth of lesions, and anatomic variation of the segmental structures often occur, which makes it difficult to perform precise segmentectomy and achieve radical resection. In our study, a $3 \mathrm{D}$ digital reconstruction system was used to reveal the relationship between the lesions and intra-pulmonary vessels and bronchi, and to obtain anatomic information on the whole-lung volume, lesion volume, location, and length and diameter of each structure. Individualized segmentectomy was planned, with consideration of the proper tumor margin. Resection planes were designed during preoperative virtual surgery.

According to previous reports, pulmonary complications after pulmonary segmentectomy ranged from $6.6 \%$ to $17.3 \%(4,19)$. Additionally, persistent air leak or delayed pneumothorax after segmentectomy is prevalent and increases surgical morbidity (20). Previous reports also 
Table 3 Clinical, operative characteristics of patients in the non-CDR and CDR groups

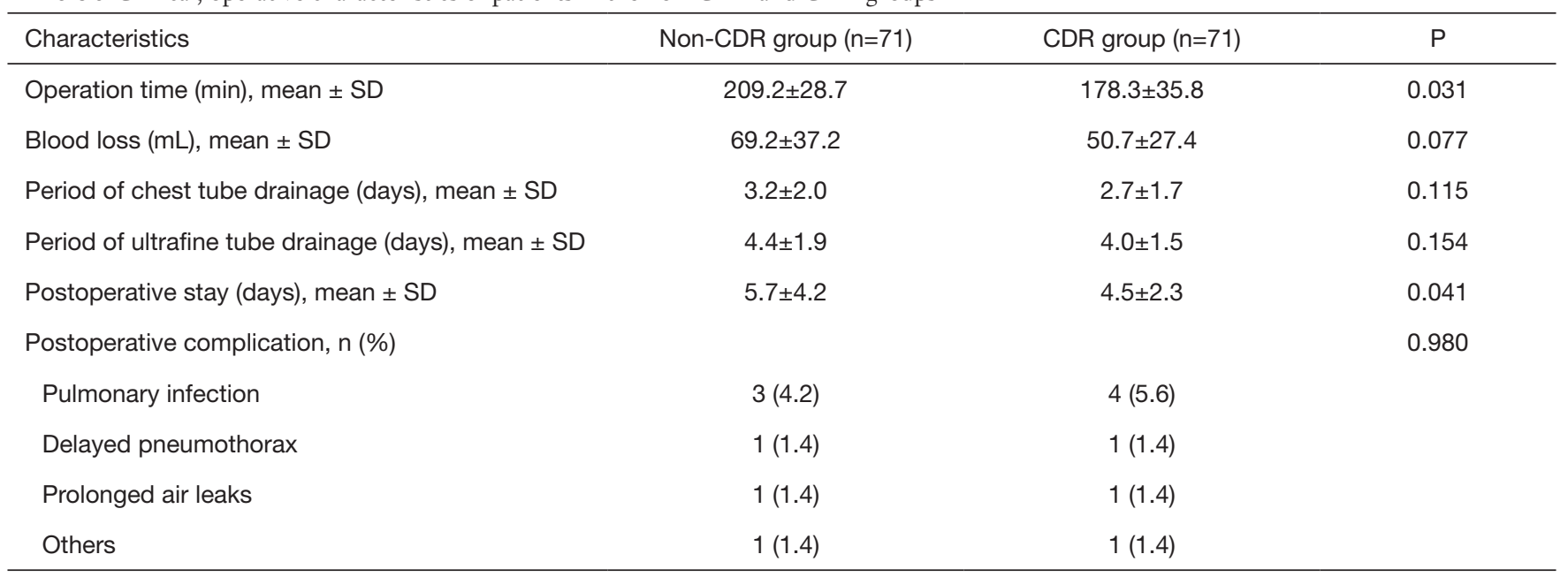

CDR, Combined Dimensional Reduction Method; SD, standard deviation.
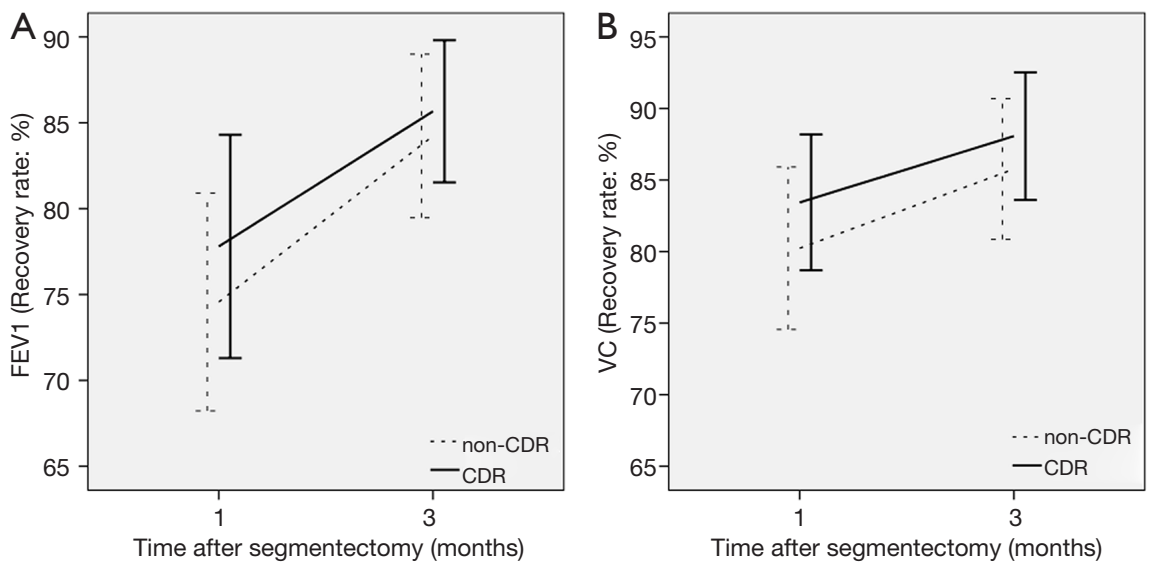

Figure 3 Pulmonary function after segmentectomy. (A) Forced expiratory volume in 1 second (FEV1) at 1 and 3 months after segmentectomy in the non-CDR group (regular line) and CDR group (spotted line). (B) Vital capacity (VC) at 1 and 3 months after segmentectomy in the non-CDR group (regular line) and CDR group (spotted line). The y-axis shows the recovery rate. Error bars are expressed as mean $\pm \mathrm{SD}$. CDR, Combined Dimensional Reduction Method.

suggested that the incidence of delayed pneumothorax ranged from $2.2 \%$ to $18 \%(20,21)$. Several methods to prevent air leak from the opening of ISP after segmentectomy were evaluated $(4,5,20,21)$. The advantage of the pleural suture method, which includes mechanical stapling, is the reduction of the risk for postoperative pulmonary complications. However, other studies have indicated that it might cause shrinkage of the preserved segment, and so the influence of the method on the postoperative pulmonary function is still controversial $(3,12,20)$. When a surgical stapler is used, it is difficult to identify the preserved intersegmental vein during dissection accurately. Performing a less extensive resection might cause insufficient surgical margins. Extension of the resection to the adjacent segment may injure the marginal vein and lead to congestion in the residual segment (20). Several studies have reported intraoperative blood loss, and postoperative air leak was almost similar between intersegmental dissection with electrocautery and the stapler $(4,20)$. However, eschars caused by the application of electrocautery make it more difficult to distinguish the ISP.

To the best of our knowledge, our study is the first study 
to report on the combined usage of ultrasonic scalpel and stapler for management of ISP. As mentioned earlier, both staplers and electrocautery have been used extensively in ISP management, but despite their strengths, both have their respective drawbacks. Ohtsuka et al. reported on the comparison between the combined use of electrocautery and staplers against the use of electrocautery alone. They found that the use of staplers in addition to electrocautery may lead to the reduction of prolonged air leak (4). The effect of ultrasonic scalpels for management of ISP is still under debate. Matsumura et al. who suggest the possible superiority of this method, firstly reported the surgical technique of pulmonary segmentectomy by ultrasonic scalpel for the dissection of the ISP associated with absorbable sealing materials to cover the cut surface of the lung (22). However, Takagi et al. had reported that the incidence of the late onset of a pulmonary fistula was higher when a harmonic scalpel was used, as compared with electrocautery (21). In the current study, the use of ultrasonic scalpel and staplers for management of ISP was associated with the shorter operating time and postoperative stay, with no significant differences in the period of tube drainage as well as the incidence of postoperative complication than the conventional non-CDR method. Thus, we believe that the combined use of ultrasonic scalpel and stapler is an effective method with acceptable postoperative complications. Moreover, the CDR method was also superior in terms of the cost-effectiveness, as the reducing numbers of using staplers and the shorter postoperative hospital stay.

Those 4 parameters of age, gender, the number of cigarette packs smoked per year and predicted FEV1 for propensity matching seems not correlated to some of the outcomes. The parameters are one of the limitations of our study, and some better markers should be applied in the future. However, we had controlled the potential variables as far as possible, including that the same group of surgeons conducted all of the surgeries in this study, and after matching. There was no significant difference in the observed variables of clinical characteristics, such as age, gender, tumor size, type of segmentectomy and pathological diagnosis. Thus we believe it is receivable to conduct the comparisons. Besides, our research also limited in its retrospective nature and the limited numbers of enrolled patients. Overall, however, the bulk of evidence indicates that the CDR method could aid in facilitating the ease and precision of uniport VATS segmentectomy, and has the potential to be an efficacious and practical technique for pulmonary segmentectomy

\section{Acknowledgments}

Funding: The study was supported by the Medical Innovation Project of Fujian Health and the Family Planning Commission (2015-CX-19), Fujian Provincial Major Special Projects (2017YZ0101), and Provincial Science and Technology Leading Project (2018Y0032).

\section{Footnote}

Conflicts of Interest: The author has no conflicts of interest to declare.

Ethical Statement: This study was approved by the ethics committee of Fujian Medical University Union Hospital (No. 2018020). Informed consent obtained from the patients after discussion of the general risks and benefits of pulmonary segmentectomy. The authors are accountable for all aspects of the work in ensuring that questions related to the accuracy or integrity of any part of the work are appropriately investigated and resolved.

\section{References}

1. Saghir Z, Dirksen A, Ashraf H, et al. CT screening for lung cancer brings forward early disease. The randomised Danish Lung Cancer Screening Trial: status after five annual screening rounds with low-dose CT. Thorax 2012;67:296-301.

2. Yao F, Wang J, Yao J, et al. Reevaluation of the efficacy of preoperative computed tomography-guided hook wire localization: A retrospective analysis. Int J Surg 2018;51:24-30.

3. Yoshimoto K, Nomori H, Mori T, et al. Comparison of postoperative pulmonary function and air leakage between pleural closure vs. mesh-cover for intersegmental plane in segmentectomy. J Cardiothorac Surg 2011;6:61.

4. Ohtsuka T, Goto T, Anraku M, et al. Dissection of lung parenchyma using electrocautery is a safe and acceptable method for anatomical sublobar resection. J Cardiothorac Surg 2012;7:42.

5. Matsumoto M, Shirahashi K, Yamamoto H, et al. Division of the intersegmental plane using electrocautery for segmentectomy in clinical stage I non-small cell lung cancer. J Thorac Dis 2018;10:S1215-21.

6. Wang J, Xu X, Wen W, et al. Modified method for 
distinguishing the intersegmental border for lung segmentectomy. Thorac Cancer 2018;9:330-3.

7. Iwata H, Shirahashi K, Mizuno Y, et al. Surgical technique of lung segmental resection with two intersegmental planes. Interact Cardiovasc Thorac Surg 2013;16:423-5.

8. Zeiher BG, Gross TJ, Kern JA, et al. Predicting postoperative pulmonary function in patients undergoing lung resection. Chest 1995;108:68-72.

9. Lex JR, Naidu B. In patients with resectable nonsmall-cell lung cancer, is video-assisted thoracoscopic segmentectomy an appropriate alternative to videoassisted thoracoscopic lobectomy? Interact Cardiovasc Thorac Surg 2016;23:826-31.

10. D'Andrilli A, Rendina EA. POINT: Should Segmentectomy Rather Than Lobectomy Be the Operation of Choice for Early-Stage Non-small Cell Lung Cancer? Yes. Chest 2018;153:590-2.

11. Nguyen D, Gharagozloo F, Tempesta B, et al. Long-term results of robotic anatomical segmentectomy for earlystage non-small-cell lung cancer. Eur J Cardiothorac Surg 2019;55:427-33

12. Nomori H, Shiraishi A, Cong Y, et al. Differences in postoperative changes in pulmonary functions following segmentectomy compared with lobectomy. Eur J Cardiothorac Surg 2018;53:640-7.

13. Wu CF, Fernandez R, de la Torre M, et al. Midterm survival outcome of single-port video-assisted thoracoscopic anatomical lung resection: a two-centre experience. Eur J Cardiothorac Surg 2018;54:252-9.

14. Xiao F, Yu Q, Zhang Z, et al. Novel perspective to evaluate the safety of segmentectomy: clinical significance of lobar and segmental lymph node metastasis in cT1N0M0 lung adenocarcinoma. Eur J Cardiothorac

Cite this article as: Zheng $\mathrm{B}, \mathrm{Xu} \mathrm{G}, \mathrm{Fu} \mathrm{X}, \mathrm{Wu} \mathrm{W}$, Liang $\mathrm{M}$, Zeng T, Zhang S, Zhu Y, Zheng W, Chen C, Bédat B, Swanson SJ, Koike T, Iwata H, Bedetti B, Sato M; written on behalf of the AME Thoracic Surgery Collaborative Group. Management of the inter-segmental plane using the "Combined Dimensional Reduction Method" is safe and viable in uniport video-assisted thoracoscopic pulmonary segmentectomy. Transl Lung Cancer Res 2019;8(5):658-666. doi: 10.21037/tlcr.2019.09.12
Surg 2018;53:228-34.

15. Nardini M, Bilancia R, Paul I, et al. (99m)Technetium and methylene blue guided pulmonary nodules resections: preliminary British experience. J Thorac Dis 2018;10:1015-21.

16. Okusanya OT, Hess NR, Luketich JD, et al. Infrared intraoperative fluorescence imaging using indocyanine green in thoracic surgery. Eur J Cardiothorac Surg 2018;53:512-8.

17. Iguchi T, Yoshioka T, Muro M, et al. Systemic air embolism during preoperative pulmonary marking with a short hook wire and suture system under CT fluoroscopy guidance. Jpn J Radiol 2009;27:385-8.

18. Sakiyama S, Kondo K, Matsuoka H, et al. Fatal air embolism during computed tomography-guided pulmonary marking with a hook-type marker. J Thorac Cardiovasc Surg 2003;126:1207-9.

19. Schuchert MJ, Abbas G, Awais O, et al. Anatomic segmentectomy for the solitary pulmonary nodule and early-stage lung cancer. Ann Thorac Surg 2012;93:1780-5; discussion 1786-7.

20. Saito H, Konno H, Atari M, et al. Management of Intersegmental Plane on Pulmonary Segmentectomy Concerning Postoperative Complications. Ann Thorac Surg 2017;103:1773-80.

21. Takagi K, Hata Y, Sasamoto S, et al. Late onset postoperative pulmonary fistula following a pulmonary segmentectomy using electrocautery or a harmonic scalpel. Ann Thorac Cardiovasc Surg 2010;16:21-5.

22. Matsumura Y, Okada Y, Shimada K, et al. New surgical technique of pulmonary segmentectomy by ultrasonic scalpel and absorbable sealing materials. Kyobu Geka 2004;57:31-7. 